\title{
Design and Control Recipes for Complex Photonic Integrated Circuits
}

\author{
Maziyar Milanizadeh
}

\section{Introduction to Photonic Integrated Circuits}

In the last decades optical communications contributed to the huge diffusion of the telecommunication market, pushing the development of new technologies to enable higher performances and lower costs. We are witnessing the introduction of the light at every level of the communication network. Long distance links, national backbones and metropolitan networks are equipped with optical fibers that guarantees higher performances and lower costs than older technologies, e.g. radio or coaxial links. In Europe, U.S. and Japan there are projects to introduce fibers also in the short distance links (Next Generetion Access Network) ensuring a great rise of the bandwidth for the final consumer user. Optical bus will be introduced in the next future in the rack backplanes, on the boards and finally also into the chips on the boards, joining electronic devices and substituting them in the information delivering.

Then the photonic technologies are in a very predominant position in the today telecommunication scenario, that becomes the driving force behind the development of photonic integration. For example, optical components become the enabling factors for the optical networks when a complexity enhancement is required (e.g. where there is the necessity to move from point-to-point links to more complex network structures, introducing advanced all-optical switching technologies). Obviously not only telecommunication area takes advantage of this progress in technology but also different fields such as sensor for physical, medical or civil monitoring.

Photonic integration has a very long history dating back to the '70s. Starting from the first products the trend continued with more complicated structures bringing, in the present day, to the possibility to implement complex functions in very small chips, following more or less the path traced by the electronics some decades before. But at the moment the research on optical components is late compared with the market

\footnotetext{
M. Milanizadeh ( $\square)$

Dipartimento di Elettronica, Informazione e Bioingegneria, Politecnico di Milano, Via Ponzio 34/5, 20133 Milano, Italy

e-mail: maziyar.milanizadeh@polimi.it

(C) The Author(s) 2022

L. Piroddi (ed.), Special Topics in Information Technology,

PoliMI SpringerBriefs, https://doi.org/10.1007/978-3-030-85918-3_12
} 
requests, which show the necessity to integrate a lot of functions on a single chip with very low costs. This generate the great push toward the photonic integration, in particular for PIC with high complexity (in terms of number of components per PIC).

Nowadays, integrated photonics technologies are envisioned as fundamental for applications such as optical communications [1-3], optical interconnects [4-6], bio-sensing [7-9], 5G networks [10, 11] and quantum photonics [12].

Among all the technological platforms that can be used to realize PIC, two semiconductors technologies have been emerging in the last decade: Indium Phosphide and Silicon. Thanks to the high index contrast offered by these photonic platforms, it is possible to integrated a large number of devices on the same chip and to implement complex functionalities for the generation, manipulation and detection of light. Indium phosphide offers the possibility of monolithically integrating on-chip waveguide, detectors, modulators and light sources [13], while silicon allows an unprecedented number of integrated components [14].

To reach the objective of replacing electronic circuits with low power consumption photonic circuits, especially for telecom/datacom applications [15, 16], it is necessary to handle more and more complex functions in the optical domain [17]. To realize such complex functions, photonic circuits must reach an higher level of complexity, interconnecting many photonic devices on the same chip.

Despite the device miniaturization achievable with silicon photonics technologies [18], the integration of these devices is a separated issue [19] that must be challenged to deliver advanced functionalities on photonic integrated chips [18]. This scaled complexity must be matched with the urgent needs of adaptability and programmability to enable the realization of arbitrary, reconfigurable, complex circuits thus shifting the paradigm from a device-level to a "system-on-a-chip" one.

\subsection{The Control Paradigm}

The extreme device miniaturization reached by state-of-the art photonic technologies now enables the realization of hundreds or even thousands of photonic elements in a footprint of less than $1 \mathrm{~mm}^{2}$ [14]. Although many building blocks potentially provide the required degrees of freedom to realize flexible and arbitrarily complex photonic architectures, reconfigurable optical circuits aggregating many different functionalities are still encountering strong difficulties to emerge. The reason is that in photonics, similarly to electronics, device miniaturization is not synonymous with large scale of integration, and some keys still need to be found to make photonics step up from the current device level to complex, adaptive and reconfigurable integrated circuits.

In other side, PICs are evolving towards on-chip re-configurable architectures and general purpose programmable photonic processors, enabling the implementation of many different functionalities on-demand [20-23]. These schemes rely on the use of 
a large number of optical interferometers, such as MZI and MRRs, whose individual working point is inherently related to the phase delay between the interfering optical beams. Therefore, any kind of phase perturbation may substantially affect the overall behavior of the PIC.

To reach these goals, feedback control is mandatory to steer and hold the entire system to the desired functionality, and make it immune to fabrication tolerances, functional and environmental drifts, and mutual crosstalk effects. In fact sensitivity to temperature fluctuations is one of the strongest limiting factors to the exploitation of integrated optical devices. This effect is particularly relevant in Silicon on Insulator (SOI), where the large thermo-optic coefficient (TOC) of silicon $\left(1.8 * 10^{-4} \mathrm{~K}^{-1}\right.$ at $300 \mathrm{~K}$ [25]) is responsible for a wavelength shift of any interference-based device of about $10 \mathrm{G} \mathrm{Hz} \cdot \mathrm{K}^{-1}$. In interferometric devices, a waveguide width deviation of only $1 \mathrm{~nm}$ can produce a frequency shift of about $100 \mathrm{GHz}$ in the spectral response [24]. Due to this sensitivity, and to the tolerances of current fabrication technologies, the response of fabricated PIC hardly matches the design performance; moreover, it is possible that reconfiguration capabilities will be needed to adapt in real time the circuit to new requirements (i.e. dynamic switching and routing, channels add/drop and so on). Due to functional drifts and components ageing over time, is not possible to have an accurate and robust control only relying on lookup tables; hence, there is a need of an automatic feedback loop to set the new working point automatically and to control real time the PIC to counteracts unwanted drifts.

The paradigm of a feedback loop for integrated photonics is shown in Fig. 1. One or more detectors are placed in strategic positions throughout the circuit; these detectors, which can be fast or slow depending on what is being measured, generate control signals proportional to the measure they are performing. A controller reads the control signals and estimates the working point of the PIC based on the information provided; the controller then drives the actuators to steer the working point accordingly to the algorithm implemented in the controller itself. The actuators rely on physical effects to modify the working point of the PIC. Conveniently, control systems should be low cost, energy efficient, insensitive to fluctuations of the optical power, applicable to both passive and active devices, and should not require additional photonic structures.

Optical Design and control approaches should bind together for development of complex reconfigurable PIC. This means along the design process we need to take

Fig. 1 Closed loop control of PIC. The signals from detectors placed in strategic positions throughout the circuits is used by a controller to estimate the current working point. Actuators are then driven to modify the working point of the circuit accordingly to the logic implemented in the controller

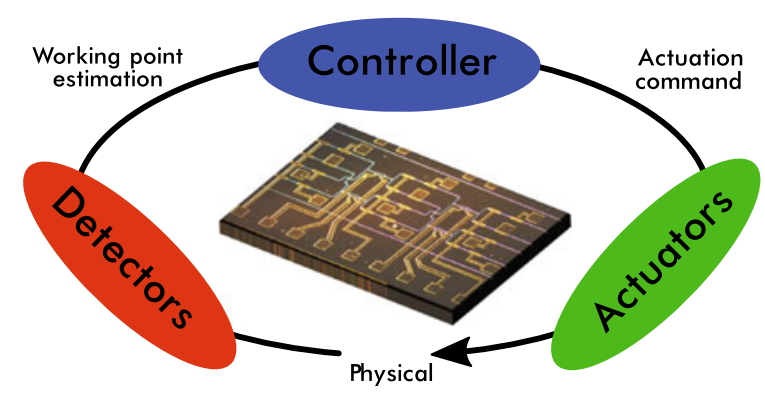


into account tunability of the device in different scenario of application and for variety of possible perturbations. In this work we study some of these techniques and introduce new approaches to design and control complex PICs, reconfigurable along the operation band.

\section{Key Proposed Innovative Concepts}

The work reported in this thesis has outcomes in diverse areas under the Integrated Photonics umbrella. Solutions and techniques from design to calibration and control phase of PIC implementation are discussed and analyzed through numerical simulations and experimental trials of different functions implemented in multiple technologies.

\section{- Thermal crosstalk free system}

We discuss extensively the idea of cross talk between effect of actuators in PIC. For the example of thermo-optic actuators, due to heat flow from actuator to other parts of chip, perturbation is introduced in unwanted places of circuit from each actuator. To mitigate this perturbations other heaters need to change their working point which lead to other perturbations which in some cases can cause instability in control algorithms. Several solutions have been proposed to mitigate thermal crosstalk on a photonic chip. Thermal isolation trenches allow the localization of the heat around the actuated waveguide and can also improve the heater efficiency [21, 26]. However, this approach puts some constraints on the layout and footprint of the PIC. In this work we suggest instead of individual modifications of actuators, they are modified by specific weights which are obtained from eigen-solution of the coupled system (introduced as Thermal eigenmode decomposition, TED). Through numerical simulations and experimental trials we examine the efficiency of this technique. We suggest an effective technique to evaluate coupled system to deliver the optimum cross-talk cancellation with TED based techniques.

A key point of the TED method is the knowledge of the $\mathbf{T}$ matrix describing the thermal coupled system. In principle it could be inferred from thermal simulations. However, this approach is hardly practical for large scale PICs accounting for many heat sources, especially because the overall assembly of the PIC should be considered, including on-chip metal lines, wire bonding, chip submount and ultimately the package itself. Therefore, for an accurate estimation of the $\mathbf{T}$ matrix, alternative strategies should be adopted, which should be preferably applicable to arbitrary PIC architectures. 
We considered three different solutions to evaluate the thermal crosstalk with different accuracy:

1. Heuristic (lowest accuracy): an estimation of thermal cross talk is inferred by simply considering the topology of the circuit;

2. Optical measurement (highest accuracy, not always practicable): thermal crosstalk is directly estimated by measuring the unwanted wavelength shift of neighbor devices;

3. Electrical measurement (good accuracy, always practicable): thermal crosstalk is indirectly estimated by measuring the change of the electric resistance of neighbor heaters used as temperature probes;

It is worth to mention that $\mathbf{T}$ depends only on the device architecture and not on the specific operation and, as any transfer function, must be evaluated once, independently on the circuit working point.

A direct optical measurement of thermal crosstalk is not possible in most cases, because it requires the possibility to optically access the output ports of each tunable element. A good estimation of the $\mathbf{T}$ matrix can be achieved electrically, by measuring the temperature-induced change of the resistance of metallic probes located in suitable spots of the PICs. To this aim, we can advantageously use the heater structures themselves, which can be used also as temperature sensors to map thermal crosstalk across the photonic chip. We managed to demonstrate through experimental trials the effectiveness of this technique. In Fig. 2 we compare of convergence efficiency of TED based control algorithms with different techniques of estimation for $\mathbf{T}$ matrix for a cross-bar connect of 4 MRRs.

Details of these techniques can be found in published work [27, 29].

\section{- Tuning to signal spectrum}

After introducing an effective techcnique to mitigate thermal cross-talk effects in PIC, we continue by discussing a new approach in tuning algorithm to identify the progress of the algorithm. Traditionally, PIC tuning is implemented targeting a specific frequency response. This approach can be valuable for testing, and pre-calibration procedures, but it is not practical for automated tuning of the PIC during its operation because it is resource and time consuming. For instance, it may require a tunable source and/or a spectrum analyzer to monitor the response at wavelengths of interest. Likewise, time domain approaches, based on the measurement of the Bit error rate (BER) or eye diagram distortion, require considerable load of Digital Signal Processor (DSP) as well as electrical power consumption. In any case both approaches are hardly practical when the device is in use. In this work we suggest using the Power Spectrum Density (PSD) of the channel as 
merit in tuning algorithm and we demonstrate through experimental trials we can tailor the frequency response of the filter, even if its not design to be modified, to the PSD of the channel, finding the best filter tuning condition for that specific channel. Using the optical power at the output of a golden filter (filter with acceptable frequency behavior) we can tune the same family of devices. In other words replication of filter frequency response which can save time and resource in calibration phase of PIC implementation. Through numerical simulations and experimental trials we demonstrated the effectiveness of this technique in tuning and tailoring PIC to different channels. Eventually, we discuss extension of this idea forcing devices to operate on specific channel through Wavelength Division Multiplexing (WDM) grid via labeling technique. Details can be found in [28, 29].

\section{- Automatic calibration and dynamic look up tables}

Based on the concept of using signal optical power in tuning and filter replication, we introduce dynamic Look Up Tables (LUT) which can be automatically created for arbitrary PIC. They can be updated due to new requirements of the operation or new conditions of the chip like perturbations from neighboring circuits. Traditionally PICs are kept in their optimum working point through pre-defined tables which are expensive to produce during the calibration of the device. These tables need to be updated due to aging of the device or new requirement of the operations. We suggest adopting TED-based algorithms while using optical power of the channel to automatically create these tables and through locking algorithms these LUTs are updated matching new conditions of operations.

\section{- Reduction of electrical I/O}

Electrical bonding pads, responsible for electrical I/O occupy huge area of photonic chips, their size can not be decreased due to mechanical limits of wire bonding techniques [30]. This dictates complex assembly techniques for large PICs like flip chip [31] approaches which other than being expensive have their limitations. In this work, we suggest two approaches to reduce the electrical connection needed to operate and control PICs. We discuss implementing electronic multiplexer in optical chip to reduce needed electrical lanes as the first solution and grouping together optical waveguides and use common detector as the second one. In this approach the sum of optical power from monitor ports of PIC is measured and needed to be carried out of the chip. Labeled signals can be used to distinguish through a demodulation of the measured signal at the controller side out of the photonic chip.

\section{- FSR free and hitless-tunable Polarization-Diversity filter}

We introduce a fully-reconfigurable add-drop filter based on high order coupled MRR with exceptional features suitable for dynamic bandwidth allocation in core networks, back-haul networks, intra- and inter-datacenter interconnects. Implemented in Silicon on Insulator platform, we recorded spurious free Free Spectrum 
Range (FSR) on 100nm wavelength range centered in $\mathrm{C}$ band in both Drop and Through port. This is achieved using non-integer ratio Vernier scheme implemented through optimization techniques to design appropriate radius of MRR and their couplers. This device can be reconfigured transparently (hitless tuning) on this wide operation band in nano-second time scale without introduction of any perturbation ( $>35 \mathrm{~dB}$ channel isolation). This feature is obtained by implementing Variable Optical Attenuators (VOA) in middle MRR via P-i-N junctions. This filter is implemented in polarization diversity scheme $(1.2 \mathrm{~dB}$ polarization dependent loss), demonstrating polarization transparency for the hole operation band. Its performance is evaluated in experimental trials specifically while adding and dropping two double polarization channels (100 Gbit/s (QPSK) and $200 \mathrm{Gbit} / \mathrm{s}$ (16-QAM)) with complex modulation scheme demonstrated in Fig. 3. Multiples of this filter can be implemented to operate on the same optical bus to add and drop channels from Dense Wavelength Division Multiplexing (DWDM) grid with operation range wider than extended $\mathrm{C}$ band. More information about this work can be found in [32].

\section{- Free space beam manipulation}

This work is concluded by discussing the integrated meshes based on MZI units. We demonstrate manipulation of free-space optical beams by using a $4 \times 1$ selfconfiguring Silicon photonic mesh. In particular, after comparing variety of integrated mesh structures a self-converging control loop is suggested and examined capable of tuning and re-steering such devices. The performance of control approach is examined for the case of global detector (single PD) or spread detection (detector at each stage). Effect of these control algorithms on the radiation performance of the device are analyzed to understand the silver lining between sensitivity and perturbation. Then using a simple $4 \times 1$ diagonal mesh, we successfully performed beam coupling from a light source in the free space to a SMF just accurately tuning the MZIs mesh. As expected, also the reversed process, from a SMF to a specific position, worked as well. Steering the beam through mesh tuning is therefore a straightforward consequence of the alienable beam coupler when used in the opposite propagation direction. Capability of mesh to reconstruct the beam front was putted to trials by introducing known and unknown (replicating unwanted free space perturbations) perturbing mediums in the image and far filed plane of the device. In all the trials (within the degrees of freedom offered by this mesh) ideal beam pattern was recovered which demonstrated the possibility of mesh to reconstruct the optimum free space channel. Integrated meshes can be automatically tuned (by simply minimizing stages) to manipulate both phase and amplitude of the array elements to offer more degrees of modification in beam front comparing with phase array structures. 


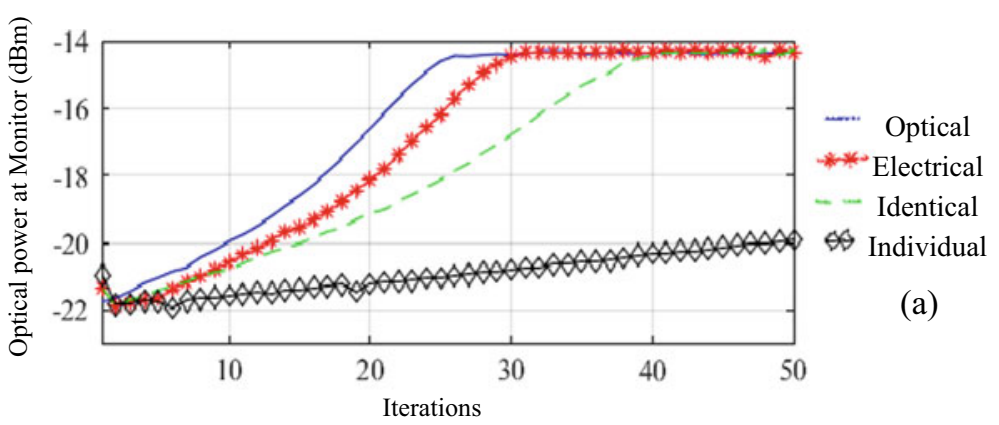

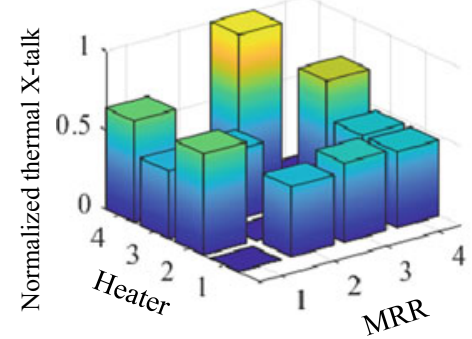

(b)

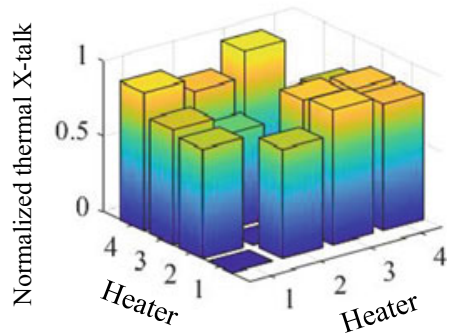

(c)

Fig. 2 a Convergence rate of TED-based tuning of the $2 \times 2$ MRR cross-bar interconnect when the off-diagonal terms of the phase coupling matrix $\mathrm{T}$ are assumed to be identical (green dashed), when they are estimated from electrical measurements (red asterisks), from optical measurements (blue solid). Black diamonds show the converge rate when MRRs are individually tuned (no-TED). b Optically and $\mathbf{c}$ electrically measured off-diagonal terms of the phase coupling matrix T normalized to the maximum value

Comparing with phase array antennas with these meshes not only an straight forward power minimization automatic tuning is achievable, due to the access to array elements amplitude, these circuits can obtain more sophisticated free space beams and introduce vast level of correction. The performance of the mesh in steering, coupling and identifying free-space beams can be improved by optimizing the design of the radiating elements, whose number can be scaled up without impairing the progressive self-configuration procedure employed for the tuning of the mesh. Applications are envisioned to more advanced free-space optical processing, including phase front reconstruction, beaming through scattering media and chip-to-chip free space communications. Details of these works can be found in [33-35]. 

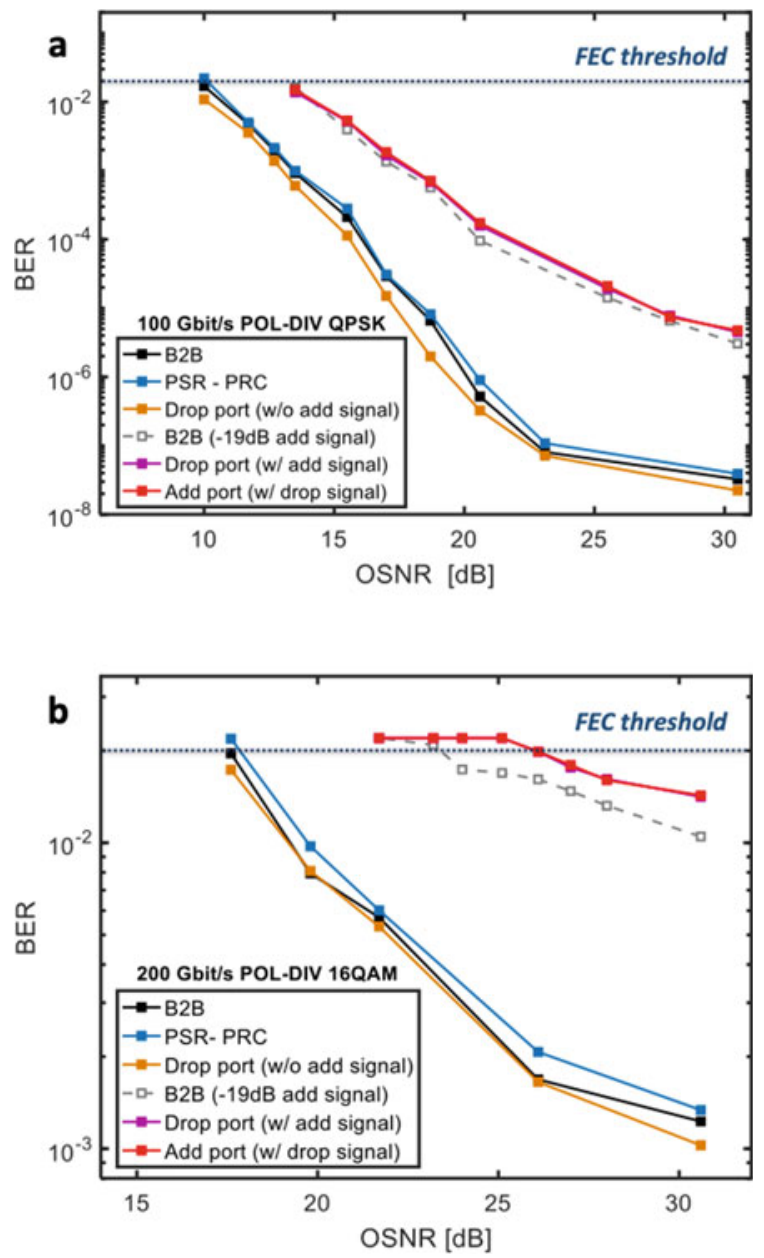

Fig. 3 BER versus Optical Signal to Noise Ratio (OSNR) of a $100 \mathrm{Gbit} / \mathrm{s}$ Pol-Div QPSK channel, b $200 \mathrm{Gbit} / \mathrm{s}$ Pol-Div 16QAM channel. Black curve for back to back scenario replacing the device with a direct fiber in the setup, Blue curve for connected device while the filters were de-tuned from the channel to measure the transparency of Pol-Div scheme, Yellow curve for dropped channel. Red and Pink curves are for adding and dropping of two channels at the same wavelength with the same modulation scheme being launched at In and ADD port. Dashed-Black curve is the performance of the transceiver for B2B condition when two channels are coupled by a fiber coupler and one (the perturbing channel) is $19 \mathrm{~dB}$ below the measuring one 


\section{References}

1. C.R. Doerr, Proposed architecture for MIMO optical demultiplexing using photonic integration. IEEE Photonics Technol. Lett. 23, 1573-1575 (2011)

2. D.A.B. Miller, Reconfigurable add-drop multiplexer for spatial modes. Opt. Express 21, 20220-20229 (2013). https://doi.org/10.1364/OE.21.020220

3. N.K. Fontaine, C.R. Doerr, M.A. Mestre, R.R. Ryf, P.J. Winzer, L.L. Buhl, Y. Sun, X. Jiang, R. Lingle, Space-division multiplexing and all-optical MIMO demultiplexing using a photonic integrated circuit, in OFC/NFOEC (2012)

4. D.A.B. Miller, Device requirements for optical interconnects to CMOS silicon chips. PMB.3, in Integrated Photonics Research, Silicon and Nanophotonics and Photonics in Switching, Optical Society of America (2010). https://doi.org/10.1364/PS.2010.PMB3

5. P. Chaisakul, D. Marris-Morini, J. Frigerio, D. Chrastina, S. Rouifed, S. Cecchi, P. Crozat, G. Isella, L. Vivien, Integrated germanium optical interconnects on silicon substrates. Nature Photonics 8, 482-488 https://doi.org/10.1038/nphoton.2014.73

6. L.-W. Luo, N. Ophir, C. Chen, L. Gabrielli, C. Poitras, K. Bergmen, M. Lipson, WDMcompatible mode-division multiplexing on a silicon chip. Nature Commun. 5 (2014) https:// doi.org/10.1038/ncomms4069

7. T. Claes, W. Bogaerts, P. Bienstman, Experimental characterization of a silicon photonic biosensor consisting of two cascaded ring resonators based on the Vernier-effect and introduction of a curve fitting method for an improved detection limit. Opt. Express 18, 22747-22761. https://doi.org/10.1364/OE.18.022747

8. M. Iqbal, M. A. Gleeson, B. Spaugh, F. Tybor, W.G. Gunn, M. Hochberg, T. Baehr-Jones, R.C. Bailey, L.C. Gunn, Label-free biosensor arrays based on silicon ring resonators and high-speed optical scanning instrumentation. IEEE J. Selected Topics Quantum Electronics 16, 654-661 (2010)

9. V. Passaro, M. La Notte, B. Troia, L. Passaquindici, G. De Francesco, G. Giannoccaro, Photonic structures based on slot waveguides for nanosensors: state of the art and future developments. Int. J. Res. Rev. Appl. Sci. 11, 411-427 (2012)

10. R. Waterhouse, D. Novack, Realizing 5G: microwave photonics for 5G mobile wireless systems. IEEE Microwave Mag. 16, 84-92 (2015)

11. J. Capmany, P. Muñoz, Integrated microwave photonics for radio access networks. J. Lightwave Technol. 32 (2014)

12. J. Silverstone, D. Bonneau, K. Ohira, N. Suzuki, H. Yoshida, N. Iizuka, M. Ezaki, C. Natarajan, M. Tanner, R. Hadfield, V. Zwiller, G. Marshall, J. Rarity, J. O'Brien, M. Thompson, On-chip quantum interference between silicon photon-pair sources. Nature Photonics 8 (2014). https:// doi.org/10.1038/nphoton.2013.339

13. M. Smit, et al., An introduction to InP-based generic integration technology. Semiconductor Sci. Technol. 29, 083001 (2014)

14. J. Sun, E. Timurdogan, A. Yaacobi, E. Hosseini, M.R. Watts, Large-scale nanophotonic phased array. Nature (2013). https://doi.org/10.1038/nature11727

15. A. Vahdat, H. Liu, X. Zhao, C. Johnson, The Emerging Optical Data Center. (2011) https:// doi.org/10.1364/OFC.2011.OTuH2

16. K. Bergman, J. Shalf, T. Hausken, Optical interconnects and extreme computing. Opt. Photon. News 27 (2016). https://doi.org/10.1364/OPN.27.4.000032

17. D.A.B. Miller, Device requirements for optical interconnects to silicon chips. Proc. IEEE 97, 1166-1185 (2009)

18. T. Baehr-Jones, T. Pinguet, P. Guo-Qiang, S. Danziger, D. Prather, M. Hochberg, Myths and rumours of silicon photonics. Nature Photonics 6, 206-208 (2012). https://doi.org/10.1038/ nphoton.2012.66

19. R. Chau, B. Doyle, S. Datta, J. Kavalieros, K. Zhang, Integrated nanoelectronics for the future. Nature Materials 6 (2007). https://doi.org/10.1038/nmat2014 
20. Y. Shen, N.C. Harris, S. Skirlo, M. Prabhu, T. Baehr-Jones, M. Hochberg, X. Sun, S. Zhao,H. Larochelle, D. Englund, et al., Deep learning with coherent nanophotonic circuits. Nature Photonics 11, 441-446 (2017). https://doi.org/10.1038/nphoton.2017.93

21. D. Pérez, I. Gasulla, L. Crudgington, D.J. Thomson, A.Z. Khokhar, K. Li, W. Cao, G.Z. Mashanovich, J. Capmany, Multipurpose silicon photonics signal processor core. Nature Commun. 8 (2017). https://doi.org/10.1038/s41467-017-01529-w

22. J. Carolan, C. Harrold, C. Sparrow, E. Martín-López, N.J. Russell, J.W. Silverstone, P.J. Shadbolt, N. Matsuda, M. Oguma, M. Itoh, G.D. Marshall, M.G. Thompson, J.C.F. Matthews, T. Hashimoto, J.L. O'Brien, A. Laing, Anthony, Universal linear optics. Am. Assoc. Adv. Sci. (2015) https://doi.org/10.1126/science.aab3642

23. L. Zhuang C.G.H. Roeloffzen, M. Hoekman, K.-J. Boller, A.J. Lowery, Programmable photonic signal processor chip for radiofrequency applications. Optica 2, 854-859 (2015). https:// doi.org/10.1364/OPTICA.2.000854

24. F. Xia, L. Sekaric, Y. Vlasov, Ultracompact optical buffers on a silicon chip. Nature Photonics. https://doi.org/10.1038/nphoton.2006.42

25. J. Komma, C. Schwarz, G. Hofmann, D. Heinert, R. Nawrodt, Thermo-optic coefficient of silicon at $1550 \mathrm{~nm}$ and cryogenic temperatures. Appl. Phys. Lett. 101 (2012). https://doi.org/ $10.1063 / 1.4738989$

26. P. Dong, W. Qian, H. Liang, R. Shafiiha, F. Ning-Ning, F. Dazeng, X. Zheng, A.V. Krishnamoorthy M. Asghari, Low power and compact reconfigurable multiplexing devices based on silicon microring resonators. Opt. Express 18, 9852-9858 (2010). https://doi.org/10.1364/ OE.18.009852

27. M. Milanizadeh, D. Aguiar, A. Melloni, F. Morichetti, Canceling thermal cross-talk effects in photonic integrated circuits. J. Lightwave Technol. 37, 1325-1332 (2019)

28. D. Aguiar, M. Milanizadeh, E. Guglielmi, F. Zanetto, G. Ferrari, M. Sampietro, F. Morichetti, A. Melloni, Automatic tuning of silicon photonics microring filter array for hitless reconfigurable add-drop. J. Lightwave Technol. 37, 3939-3947 (2019)

29. M. Milanizadeh, S. Ahmadi, M. Petrini, D. Aguiar, R. Mazzanti, F. Zanetto, E. Guglielmi, M. Sampietro, F. Morichetti, A. Melloni, Control and calibration recipes for photonic integrated circuits. IEEE J. Selected Topics Quantum Electronics 26, 1-10 (2020)

30. K. Yu, C. Chen, C. Li, H. Li, A. Titriku, B. Wang, A. Shafik, Z. Wang, M. Fiorentino, P. Yin Chiang, S. Palermo, 25Gb/s hybrid-integrated silicon photonic receiver with microring wavelength stabilization, in 2015 Optical Fiber Communications Conference and Exhibition (OFC)

31. X. Zheng, E. Chang, P. Amberg, I. Shubin, J. Lexau, F. Liu, H. Thacker, S.S. Djordjevic, S. Lin, Y. Luo, J. Yao, J.-H. Lee, K. Raj, R. Ho, J.E. Cunningham, A.V. Krishnamoorthy, A high-speed, tunable silicon photonic ring modulator integrated with ultra-efficient active wavelength control. Opt. Express 22, 12628-12633 (2014). https://doi.org/10.1364/OE.22. 012628

32. F. Morichetti, M. Milanizadeh, M. Petrini, F. Zanetto, G. Ferrari, D.O. de Aguiar, E. Guglielmi, M. Sampietro, A. Melloni, Polarization-transparent silicon photonic add-drop multiplexer with wideband hitless tuneability. Nature Commun. https://doi.org/10.21203/rs.3.rs-147585/v1

33. M. Milanizadeh, P. Borga, F. Morichetti, D.A.B. Miller, A. Melloni, Manipulating free-space optical beams with a Silicon photonic mesh, in 2019 IEEE Photonics Society Summer Topical Meeting Series (SUM)

34. M. Milanizadeh, E. Damiani, T. Jonuzi, M.J. Mencagli, B. Edwards, D.A.B. Miller, N. Engheta, A. Melloni, F. Morichetti, Recursive MZI mesh for integral equation implementation, in European Conference on Integrated Optics 2020 (ECIO)

35. M. Milanizadeh, et al, Coherent self-control of free space optical beams with integrated silicon photonic meshes, in Photonics Research, under review (arXiv:2104.08174) 
Open Access This chapter is licensed under the terms of the Creative Commons Attribution 4.0 International License (http://creativecommons.org/licenses/by/4.0/), which permits use, sharing, adaptation, distribution and reproduction in any medium or format, as long as you give appropriate credit to the original author(s) and the source, provide a link to the Creative Commons license and indicate if changes were made.

The images or other third party material in this chapter are included in the chapter's Creative Commons license, unless indicated otherwise in a credit line to the material. If material is not included in the chapter's Creative Commons license and your intended use is not permitted by statutory regulation or exceeds the permitted use, you will need to obtain permission directly from the copyright holder. 